VOL. $6(1972), 185-210$.

\title{
Inequalities related to those of Hausdorff-Young
}

\section{R.E.Edwards}

This note establishes the impossibility of certain inequalities of the form

$$
\|f\|_{p} \leq B\left(\|f\|_{p}+\|\hat{f}\|_{q}\right)
$$

holding for all trigonometric polynomials $f$ on an infinite compact abelian group $G$. From this is deduced the impossibility of corresponding inclusion relations of the type

$$
F L^{a} \subseteq U\left\{F L^{b}: b>a\right\}+U\left\{L^{c}: c<2\right\}
$$

or

$$
\cap\left\{F L^{a}: 1 \leq a<b\right\} \subseteq F L^{b}+U\left\{Z^{c}: c<2\right\},
$$

where FS denotes the Fourier image of the set $S$ of integrable functions on $G$.

\section{Introduction}

Throughout this note, $G$ denotes an infinite (Hausdorff) compact abelian group with normalised Haar measure $\lambda$, and $X$ its character group with counting measure; $L^{p}$ denotes $L^{p}(G)=L^{p}(G, \lambda)$ and $l^{p}=l^{p}(x)$. $T P=T P(G)$ denotes the set of all trigonometric polynomials on $G . \hat{f}$ denotes the Fourier transform of $f$.

The Hausdorff-Young inequality for $G$ (see [2], 13.5.1; [4],

Received 11 October 1971. The author expresses his thanks to $\mathrm{Dr}$ G.I. Gaudry for pointing out an error in the original proof of Theorem 4.2, and for other helpful comments. 
(31.22)) asserts that

$$
\|\hat{f}\|_{p^{\prime}} \leq\|f\|_{p}
$$

whenever $f \in L^{p}, 1 \leq p \leq 2$ and $p^{\prime}=p /(p-1)$. There are various senses in which this result is known to be best-possible; see, for example, [2], 13.5.3; [4], (37.19). In particular, if $1 \leq p<2$, there is no inequality of the form

$$
\|f\|_{p} \leq B\|\hat{f}\|_{p^{\prime}}
$$

valid for every $f \in T P$. (If there were, it would follow easily that $L^{p}$ would be mapped by the Fourier transform onto the whole of $\imath^{p^{\prime}}$, which is known to be false.)

Dually, the Hausdorff-Young inequality for $X$ asserts that

$$
\|f\|_{q^{\prime}} \leq\|\hat{f}\|_{q}
$$

whenever $1 \leq q \leq 2$ and $f \in T P$. Here again, if $1 \leq q<2$, there is no inequality of the form

$$
\|\hat{f}\|_{q} \leq B\|f\|_{q^{\prime}}
$$

valid for every $f \in T P$ (see again [4], (37.19)).

In this note we sharpen the above negative results by denying the possibility of inequalities of the form

$$
\|f\|_{p} \leq B\left(\|f\|_{r}+\|\hat{f}\|_{q}\right)
$$

valid for all $f \in T P$, when $p, q, r \in(0, \infty]$ satisfy certain conditions. As we shall show, the failure of an inequality (1.5) is equivalent to the failure of a corresponding inclusion relation involving vector sums of certain appropriate function spaces over $G$ or $X$. The appearance of such vector sums seems to be a novelty in this area.

DEFINITION 1.1. By a triplet we shall mean a triplet $(p, r ; q) \in(0, \infty]^{3}$. Such a triplet is said to be admissible if and only if there exists a positive number $B=B(p, r, q)$ such that (1.5) holds for every $f \in \operatorname{TP}(G)$.

A simple approximation argument shows that, if $(p, r ; q)$ is 
admissible, then (1.5) continues to hold for every continuous $f$ on $G$, and even for all $f \in L^{\max (1, r, p)}$.

In what follows, if $t \in(0, \infty], t^{\prime}$ is defined to be $\infty$, $t /(t-1), I$ according as $0<t \leq 1,1<t<\infty, t=\infty$ respectively.

1.2. We collect here a few results which are more or less immediate. Note first that, for fixed $f,\|f\|_{p}$ is an increasing function of $p$ and $\|\hat{f}\|_{q}$ a decreasing function of $q$.

(i) $(p, r ; q)$ is admissible if $p \in(0, r]$ and $q \in(0, \infty]$.

(ii) If $\left(p_{0}, r_{0} ; q_{0}\right)$ is admissible, then $(p, r ; q)$ is admissible whenever $p \in\left(0, p_{0}\right], r \in\left[r_{0}, \infty\right]$ and $q \in\left(0, q_{0}\right]$.

(iii) $(p, r ; q)$ is admissible whenever $q \in(0,2], p \in\left(0, q^{\prime}\right]$ and $r \in(0, \infty]$. (The appropriate inequality $(1.5)$ is trivially true if $q \in(0,1]$; otherwise it follows from the Hausdorff-Young inequality for $X$, that is, from (1.3).)

(iv) $(\infty, r ; q)$ is not admissible if $r \in(0, \infty)$ and $q \in(1, \infty]$. To prove (iv), take an infinite sidon set $S$ in $X$ (see [4], $(37.18))$. For $S$-spectral $f \in T P$ we have $([2], 15.14 ;[4],(37.2))$

$$
\|\hat{f}\|_{1} \leq \text { const. }\|f\|_{\infty} ;
$$

so, if $(\infty, r ; q)$ were admissible, we should have also

$$
\|\hat{f}\|_{1} \leq \text { const. }\left(\|f\|_{r}+\|\hat{f}\|_{q}\right) \text {. }
$$

But, since $S$ is Sidon, we have $([2], 15.3 .1$; [4], (37.10))

$$
\|f\|_{r} \leq \text { const. }\|f\|_{2} \text { for every } S \text {-spectral } f \in T P \text {. }
$$

Thus, by Parseval's formula, (1.6) yields

$$
\|\hat{f}\|_{1} \leq \text { const. }\left(\|\hat{f}\|_{2}+\|\hat{f}\|_{q}\right)
$$

for every $S$-spectral $f \in T P$. This signifies that

$$
\|\phi\|_{1} \leq \text { const. }\left(\|\phi\|_{2}+\|\phi\|_{q}\right)
$$

for every complex-valued $\phi$ with a finite support contained in $S$. Since $S$ is infinite and $q>1$, this is plainly false. 
1.3. From 1.2 it follows in particular that the only non-trivial cases are those in which

$$
p \in(0, \infty), r \in(0, p) \text { and } q \in(1, \infty] \text {. }
$$

A further reduction comes from the following lemma, which is an analogue of a corresponding statement about $\Lambda_{p}$-sets in $X$ (see [2], 15.5.2).

LEMMA 1.4. Suppose that $(p, r ; q)$ is admissible for at least one $r \in(0, p)$. Then $\left(p, r_{1} ; q\right)$ is admissible for every $r_{1} \in(0, p)$.

Proof. In view of 1.2 (ii), we may and will assume that $0<r_{1}<r<p$. By Hölder's inequality and the assumed admissibility of $(p, r ; q)$, we have for every $f \in T P$ satisfying

$$
\max \left(\|f\|_{r_{1}},\|\hat{f}\|_{q}\right) \leq 1
$$

the estimate

$$
\begin{aligned}
\|f\|_{r}^{r\left(p-r_{1}\right)} & \leq\|f\|_{r_{1}}^{r_{1}(p-r)}\|f\|_{p}^{p\left(r-r_{1}\right)} \\
& \leq\|f\|_{r_{1}}^{r_{1}(p-r)}{ }_{B}^{p\left(r-r_{1}\right)}\left(\|f\|_{r}+\|\hat{f}\|_{q}\right)^{p\left(r-r_{1}\right)} \\
& \leq B^{p\left(r-r_{1}\right)}\left(\|f\|_{r}+1\right)^{p\left(r-r_{1}\right)} .
\end{aligned}
$$

If we put $c=\|f\|_{p},(1.8)$ affirms that

$$
c \leq A(c+1)^{k}
$$

where $A=B^{k}$ and $k=p\left(r-r_{1}\right) / r\left(p-r_{1}\right)<1$. It follows that

$$
c \leq \max \left(1,2^{k /(1-k)} A^{1 /(1-k)}\right)=B^{\prime} \text {. }
$$

Thus

$$
\|f\|_{r} \leq B^{\prime}
$$

whenever (1.7) holds. By the homogeneity of all norms, therefore,

$$
\|f\|_{r} \leq B^{\prime}\left(\|f\|_{r_{1}}+\|\hat{f}\|_{q}\right) \text {. }
$$

Hence 


$$
\begin{aligned}
\|f\|_{p} & \leq B\left(\|f\|_{p}+\|\hat{f}\|_{q}\right) \\
& \leq B\left(B^{\prime}\|f\|_{r_{1}}+B^{\prime}\|\hat{f}\|_{q}+\|\hat{f}\|_{q}\right) \\
& \leq B^{\prime \prime}\left(\|f\|_{r_{1}}+\|\hat{f}\|_{q}\right),
\end{aligned}
$$

showing that $\left(p, r_{1} ; q\right)$ is admissible.

This lemma suggests a further definition.

DEFINITION 1.5. A pair $(p, q) \in(0, \infty]^{2}$ is termed admissible if and only if there exists $r \in(0, p)$ such that the triplet $(p, r ; q)$ is admissible - in which case $\left(p, r_{1} ; q\right)$ is admissible for every $r_{1} \in(0, p)$.

\section{The first main theorem}

This theorem falls into two parts, according as $p>1$ or $p=1$. The former case is easier to prove and is dealt with first and separately.

THEOREM 2.1. If $p>1$ and $q>2,(p, q)$ is not admissible.

Proof. This proceeds by contradiction. If the assertion were false, the triplet $(p, r ; q)$ would be admissible for some $p>1$, some $q>2$ and every $r \in(0, p)$. Hence in particular we should have

$$
\|f\|_{p} \leq B\left(\|f\|_{I}+\|\hat{f}\|_{q}\right) \text {. }
$$

for every $f \in T P$.

Let $\mu$ be a (Radon) measure on $G$ such that $\hat{\mu} \in \imath^{q}$. Apply (2.l) with $f$ replaced by $f_{j}=k_{j} * \mu$, where $k_{j}$ is an approximate identity of trigonometric polynomials satisfying $\sup _{j}\left\|K_{j}\right\|_{1} \leq 1$. We then have for every $j$

$$
\left\|f_{j}\right\|_{1} \leq\|\mu\|
$$

and

$$
\left\|\hat{f}_{j}\right\|_{q} \leq\|\hat{\mu}\|_{q} \text {. }
$$

It would follow from (2.1)-(2.3) that the numbers $\left\|f_{j}\right\|_{p}$ are bounded with respect to $j$ and so, since $p>1$, that the net $\left(f_{j}\right)$ has a weak 
limiting point $f$ in ${ }_{L}^{p}$. Since also the measures $f_{j} \lambda$ converge weakly to $\mu$, it would follow that $\mu=f \lambda$ and so that $\mu$ is absolutely continuous. It would thus appear that every measure whose Fourier. transform belongs to $\imath^{q}$ is necessarily absolutely continuous. This contradicts the proof of Theorem 5.3 in [5], which establishes the existence of a continuous singular measure on $G$ whose transform belongs to $z^{q}$ for every $q>2$.

REMARK 2.2. When $1<p<2$, this sharpens the known failure in various ways of (1.2).

The next two lemmas are used to derive the excluded case, $p=1$, of Theorem 2.1 for certain groups $G$. Whether or not the excluded case of Theorem 2.1 is valid for every infinite compact abelian $G$ seems to be an open problem.

As will appear in 2.5, both lemmas have some intrinsic interest. The first is an extension of Lemma (44.50) of [4], the notation of which is used here.

LEMMA 2.3. Suppose that $\left(U_{n}\right)$ is a D-sequence in $G$ and $\left(K_{n}\right)$ is an approximate identity such that

$$
\left\|K_{n}\right\|_{1} \leq 1,0 \leq K_{n} \leq \kappa^{\prime} \xi_{U_{n}} / \lambda\left(U_{n}\right) .
$$

Let $p \in(0,1)$ and let $\mu$ be a measure on $G$; write

$$
f_{n}=K_{n} * \mu, \quad \mu^{*}=\sup \left|f_{n}\right| \text {. }
$$

There exists a positive real number $c_{p}$, depending at most on $p,\left(U_{n}\right)$ and $K^{\prime}$, such that

$$
\left\|\mu^{*}\right\|_{p} \leq C_{p}\|\mu\|
$$

Proof. First observe that (2.4) combines with (44.50, vi) of [4] to show that

$$
\left\|\sup _{n}\left(k_{n} * g\right)\right\|_{p}^{p} \leq\left(\kappa \kappa^{\prime}\right)^{p}\|g\|_{1}^{p} /(1-p)
$$

for every $g \in L^{1}$, where $K$ is as in (44.10, ii) of [4]. 
For every positive integer $N$ define $F_{N}=\sup _{n \leq N}\left|f_{n}\right|$. Since $F_{N} \uparrow \mu^{*}$, it will suffice (Fatou's Lemma) to show that for every $N$

$$
\left\|F_{N}\right\|_{p}^{p} \leq c_{p}^{p}\|\mu\|^{p}
$$

To prove (2.7), choose and fix $N$ and a positive number $\varepsilon$. Since $\left(K_{n}\right)$ is an approximate identity, a positive integer $N^{\prime}$ can be chosen so large that

$$
\| K_{n} * K_{N^{\prime}}-\left.K_{n}\right|_{1} \leq \varepsilon / N \text { for } n \leq N
$$

Accordingly,

$$
\begin{aligned}
\left|F_{N}\right| & \leq \sup _{n \leq N}\left(K_{n} *\left|K_{N^{\prime}} * \mu\right|\right)+\sup _{n \leq N}\left(\left|K_{n}-K_{n} * K_{N^{\prime}}\right| *|\mu|\right) \\
& =\sup _{n \leq N}\left(K_{n} * g\right)+\sup _{n \leq N}\left(\left|K_{n}-K_{n} * K_{N^{\prime}}\right| *|\mu|\right)
\end{aligned}
$$

where $g=\left|K_{N^{\prime}} * \mu\right| \in L^{1}$. So, by $(2.6)$ and the assumption $p \in(0,1)$,

$$
\begin{aligned}
\left\|F_{N}\right\|_{p}^{p} & \leq\left\|\sup _{n}\left(K_{n} * g\right)\right\|_{p}^{p}+\left\|\sup _{n \leq N}\left(\left|K_{n}-K_{n} * K_{N^{\prime}}\right| *|\mu|\right)\right\|_{p}^{p} \\
& \leq(I-p)^{-1}\left(\kappa k^{\prime}\right)^{p}\left\|_{g}\right\|_{1}^{p}+\|h\|_{p}^{p},
\end{aligned}
$$

say. Now, again since $p \in(0,1)$ and $\lambda(G)=1$,

$$
\begin{aligned}
\|h\|_{p} & \leq\|h\|_{1} \\
& \leq \sum_{n \leq N}\left\|\left|K_{n}-K_{n} * K_{N^{\prime}}\right| *|\mu|\right\|_{1} \\
& \leq \sum_{n \leq N} N^{-1} \varepsilon\|\mu\| \\
& =\varepsilon\|\mu\|,
\end{aligned}
$$

the last step by (2.8). Thus

$$
\left\|F_{N}\right\|_{p}^{p} \leq(1-p)^{-1}\left(\kappa \kappa^{\prime}\right)^{p}\|\mu\|^{p}+\varepsilon^{p}\|\mu\|^{p} .
$$

If $\varepsilon$ is allowed to tend to zero, (2.7) follows, with $c_{p}=(1-p)^{-1 / p_{K K}}$.

In the following lemma, the notation is as in Lemma 2.3 , save that now 
we suppose $\left(U_{n}, V_{n}\right)$ to be a $D^{\prime \prime}$-sequence in $G$ and that the continuous functions $K_{n}$ are chosen as in (44.20) of [4].

LEMMA 2.4. Let $f$ denote the absolutely continuous part of $\mu$. Then

$$
\lim _{n \rightarrow \infty}\left\|f-K_{n} \star \mu\right\|_{p}=0 \text { for every } p \in(0,1) \text {. }
$$

Proof. By (44.22) of [4], $f_{n}+f$ almost everywhere. By Lemma 2.3, since $f \in L^{1}$,

$$
\left|f_{n}-f\right| \leq \mu^{*}+|f| \in I^{p}
$$

Thus (2.9) is a consequence of the dominated convergence theorem.

REMARKS 2.5. It is not difficult to show that the continuous functions $K_{n}$ in Lemma 2.4 could be replaced by trigonometric polynomials sharing with them all the properties listed in (44.20) of [4]. This is not essential to our application of Lemma 2.4 in the next theorem, however.

Lemma 2.4 embraces various analogues of results about Abel and (C, I) summabjlity on the circle group T; see [6], Volume I, pp. 105, 157.

The basic theorems (44.20) and (44.22) of [4], and the Lemmas 2.3 and 2.4 immediately above, seem especially interesting when compared with the results for finite products $G=T^{m}$ of the circle group given in [6], Volume II, p. 308, Theorem (2.14). In Zygmund's discussion, the single sequence $\left(K_{n}\right)$ is replaced by the multisequence $\left(K_{n}\right)$, where $\mathbf{n}=\left(n_{1}, \ldots, n_{m}\right), n_{1}, \ldots, n_{m}$ are positive integers, and

$$
K_{n}\left(\exp \left(i t_{1}\right), \ldots, \exp \left(i t_{m}\right)\right)=K_{n_{1}}\left(\exp \left(i t_{1}\right)\right) \ldots K_{n_{m}}\left(\exp \left(i t_{m}\right)\right) \text {, }
$$

each factor on the right being a one-dimensional Fejér kernel; this multisequence corresponds to multiple $(C, 1)$-summability. For the maximal function

$$
\sigma_{*} f=\sup _{n}\left|K_{n} * f\right|
$$

Zygmund's Theorem asserts that 


$$
\left\|\sigma_{*} f\right\|_{p} \leq C_{p, m}\left\{1+\int_{G}|f|\left(\log { }^{+}|f|\right)^{m-1} d \lambda\right\}
$$

for $p \in(0,1)$, while the proof shows that, if $\phi$ is any nonnegative increasing function on $[0, \infty)$ such that $\phi(u)=o\left(u \cdot \log ^{m-1} u\right)$ for large $u$, then there exists a nonnegative $f \in L^{l}$ such that $\phi \circ f \in L^{l}$ and $\sigma_{*} f(x)=\infty$ for every $x \in G$.

For the same choice of $G$, the simplest examples of our sequence $\left(k_{n}\right)$ in Lemmas 2.3 and 2.4 are such as to give rise to species of multiple Riemann summability. Inasmuch as the sequence $\left(K_{n} * \mu\right)$ and the maximal function $\mu^{*}$ are subject to (2.9) and (2.5), Riemann's method is thus seen to be in some senses more effective than the unrestricted $(C, 1)$-method, when $m>1$.

On the other hand, and a little unfortunately, even when $m=1$ the divergence of the Fejér kernel from the behaviour specified in (2.4) would seem too wide to permit a direct deduction from Lemmas 2.3 and 2.4 of the basic positive results about $(C, 1)$-summability.

THEOREM 2.6. Assume that $G$ admits at least one $D^{\prime \prime}$-sequence. Then $(1, q)$ is admissible for no $q>2$.

Proof. Assume that $q>2$ and that $(1, q)$ were admissible. Let $r \in(0,1)$. Then the triplet $(1, r ; q)$ would be admissible and so we would have

$$
\|f\|_{1} \leq B\left(\|f\|_{p}+\|\hat{f}\|_{q}\right)
$$

for every $f \in T P$ and hence also for every continuous $f$.

Take any measure $\mu$ on $G$ such that $\hat{\mu} \in \imath^{q}$ : we will deduce from (2.10) that $\mu$ is absolutely continuous, which will give a contradiction exactly as in the proof of Theorem 2.1. Indeed, write $f$ for the absolutely continuous part of $\mu$ and $f_{n}=K_{n} * \mu$, as in Lemas 2.3 and 2.4. By Lemma 2.4, $f_{n} \rightarrow f$ in $L^{r}$ and hence

$$
\left\|f_{m}-f_{n}\right\|_{r} \rightarrow 0 \text { as } m, n \rightarrow \infty \text {. }
$$


Since also $\hat{K}_{n} \rightarrow 1$ boundedly, it follows that

$$
\left\|\hat{f}_{m}-\hat{f}_{n}\right\|_{q}+0 \text { as } m, n \rightarrow \infty \text {. }
$$

Applying (2.10) with $f$ replaced by $f_{m}-f_{n}$, (2.11) and (2.12) show that $\left(f_{n}\right)$ is Cauchy in $L^{1}$. It follows that $\left(f_{n}\right)$ converges in $L^{1}$ to a limit which cannot be other than $f$ (its limit in $L^{r}$ ). Hence $\hat{f}_{n} \rightarrow \hat{f}$ pointwise on $X$. On the other hand $\hat{f}_{n}=\hat{K}_{n} \hat{\mu}$ converges pointwise on $X$ to $\hat{\mu}$, whence it results that $\hat{f}=\hat{\mu}$ and so that $\mu=f \lambda$, showing that $\mu$ is absolutely continuous. This completes the proof.

\section{The second main theorem}

The results of $\$ 2$ refer to the case $q \in(2, \infty]$; in this section we consider the remaining case $q \in(0,2]$.

THEOREM 3.1. Suppose that $q \in(0,2]$. In order that $(p, q)$ be admissible, it is necessary and sufficient that $p \in\left(0, q^{\prime}\right]$.

REMARK 3.2. Theorem 3.1 shows in particular that in (1.3) (that is, in the Hausdorff-Young inequality for $X$ ) we cannot replace $q^{\prime}$ by anything bigger; cf. [2], 13.5.3 (1).

3.3. Proof of Theorem 3.1. The sufficiency is immediate from 1.2 (iii).

Turning to the necessity, since $q^{\prime}=\infty$ for $q \in(0,1]$, it suffices to consider the case $q \in(1,2]$, a restriction which we assume hereafter.

If $(p, q)$ is admissible, Definitions 1.1 and 1.5 show that, for some $r \in(0, p)$, we have

$$
\|f\|_{p} \leq B\left(\|f\|_{p}+\|\hat{f}\|_{q}\right)
$$

for every $f \in T P$ and therefore for any $f \in L^{\max (1, r, p)}$.

We aim to show that, if $q \in(1, \infty], p \in(0, \infty]$ and $r \in(0, p)$, then (3.1) implies $p \leq q^{\prime}$. In doing this we consider separately three cases depending on the nature of $G$, namely,

(a) $G=T$, the circle group; 
(b) $G$ not totally disconnected (= not zero dimensional);

(c) $G$ totally disconnected.

(a). In this case take a small positive number $u$ and consider the function $f \in L^{\infty}(T)$ for which $f\left(e^{i t}\right)$ is 1 or 0 according as $|t| \leq \pi u$ or $\pi u<|t| \leq \pi$ respectively. Computations and simple estimates show that

$$
\|f\|_{p}=u^{1 / p},\|f\|_{r}=u^{1 / r}
$$

and

$$
\|\hat{f}\|_{q} \leq A_{q} u^{1-1 / q}
$$

On combining (3.1)-(3.3) and letting $u$ tend to zero, it appears that $1 / p \geq 1 / q^{\prime}$, that is, $p \leq q^{\prime}$, as required.

(b). In this case there exists $([4],(24.26))$ in $X$ at least one element $x_{0}$ of infinite order. If $f \in L^{\infty}(T)$ is as in $(a)$, then $f^{\prime}=f \circ x_{0} \in L^{\infty}(G)$ and

$$
\begin{gathered}
\left\|f^{\prime}\right\|_{p}=\|f\|_{p}, \quad\left\|f^{\prime}\right\|_{r}=\|f\|_{r}, \\
\left\|\hat{f}^{\prime}\right\|_{q}=\|\hat{f}\|_{q} .
\end{gathered}
$$

In fact, $X_{0}$ maps $G$ onto $T$, whence it follows (in view of the uniqueness of normalised Haar measure on $T$ ) that

$$
\int\left(g \circ x_{0}\right) d \lambda=(1 / 2 \pi) \int_{-\pi}^{\pi} g\left(e^{i t}\right) d t
$$

for every continuous complex-valued function $g$ on $T$. The same formula therefore holds for every complex-valued function $g$ on $T$ which is the pointwise limit of a uniformly bounded sequence of continuous complex-valued functions on $T$. Applying (3.6) with $g: e^{i t}+f\left(e^{i t}\right) e^{-i n t}$, where $n \in Z$, we obtain (3.4) and also the fact that

$$
\hat{f}^{\prime}\left(x_{0}^{n}\right)=\hat{f}(n)
$$


for every $n \in Z$. On the other hand, by approximating $f$ in $L^{1}(T)$ by trigonometric polynomials $f_{j},(3.6)$ applied with $g=f-f_{j}$ shows that $f^{\prime}$ is the limit in $L^{1}(G)$ of trigonometric polynomials on $G$ with spectra contained in the subgroup $x_{0}$ of $X$ generated by $x_{0}$. The spectrum of $f^{\prime}$ is thus contained in $X_{0}$, and (3.5) follows.

The conclusion $p \leq q^{\prime}$ now follows from (3.4) and (3.5) in conjunction with the preceding discussion of case (a).

(c). Finally, if $G$ is totally disconnected, there is ([4], (7.7)) a base $V_{j}$ of neighbourhoods of the identity in $G$, each $V_{j}$ being an open-closed subgroup of $G$. Since $G$ is infinite, the positive numbers $\lambda_{j}=\lambda\left(V_{j}\right)$ tend to zero. Let $f$ denote the characteristic function of $V_{j}$ and let $X_{j}$ denote the annihilator in $X$ of $V_{j}$. Direct computation shows that

$$
\|f\|_{p}=\lambda_{j}^{l / p},\|f\|_{p}=\lambda_{j}^{1 / p}
$$

Moreover, the transform of $f$ turns out to be $\lambda_{j}$ times the characteristic function of $X_{j}$, and the Parseval formula accordingly shows that the cardinal $n_{j}$ of $x_{j}$ is given by

$$
\lambda_{j}=\|f\|_{2}^{2}=\|\hat{f}\|_{2}^{2}=\lambda_{j}^{2} n_{j},
$$

so that $n_{j}=\lambda_{j}^{-1}$. Thus

$$
\|\hat{f}\|_{q}=\lambda_{j} n_{j}^{I / q}=\lambda_{j}^{I / q^{\prime}}
$$

Combining (3.1), (3.7) and (3.8) and letting $\lambda_{j}$ tend to zero, it follows again that $p \leq q^{\prime}$.

\section{Inclusion relations equivalent to admissibility}

It is possible, without reference to the results of $\$ 2$ and $\$ 3$, to express admissibility of a triplet $(p, r ; q)$ via an inclusion relation between function spaces over $G$ or over $X$. We do precisely this in 
Theorem 4.2 and then use the results of $\S 2$ and $\S 3$ to infer that the corresponding inclusion relations are false; see Theorems 4.5 and 4.6.

The function spaces over $X$ which feature in the inclusion relations are just the Fourier images of the $L^{p}$, where $p \in[1, \infty]$; these will be denoted by $F L^{p}$. The norm on $E L^{p}$ is that for which the Fourier transformation is an isometry of $L^{P}$ onto ${ }_{F L} P$.

The appropriate function spaces over $G$ call for a little more explanation.

4.1. The spaces $P M^{k}$ of pseudomeasures on $G$. We denote by $P M=P M(G)$ the space of pseudomeasures on $G$, regarding integrable functions and (Radon) measures as being injected into $P M . P M$ is normed so that the Fourier transformation maps $P M$ isometrically onto $l^{\infty}$.

$P M$ may be identified with the dual of the space $A=A(G)$ of continuous functions with absolutely convergent Fourier series, the norm on $A$ being $\|f\|_{A}=\|\hat{f}\|_{1}$.

Those pseudomeasures having Fourier transforms in $\imath^{k}$ are the elements of the space we denote by $P M^{k}$; here $k \in(0, \infty]$. Also, $P M^{k}$ is normed so that the Fourier transformation is an isometry of $P M^{k}$ onto $z^{k}$. It thus follows that the $P M^{k}$ increase with $k$; and that $P M^{I}$ is identifiable with the space $A, P M^{2}$ with $L^{2}$, and $P M^{\infty}$ with $P M$. The Hausdorff-Young Theorem for $G$ shows that $L^{p} \subseteq P M^{p^{\prime}}$ for $p \in[1,2]$.

For future use we note the fact that, if $q \in[1, \infty]$, and if $L$ is a linear functional defined on $l^{q}$ if $q \neq \infty$ or on $c_{0}$ if $q=\infty$, L being in either case continuous for the $l^{q}$-norm, then there exists $\psi \in \tau^{q^{\prime}}$ such that

$$
L(\hat{f})=\sum_{\chi \in X} \psi(X) \hat{f}(X)=s * f(e)
$$

for every $f \in T P, s$ denoting the element of $P M^{q^{\prime}}$ whose Fourier 
transform is $\psi$, and $e$ the neutral element of $G$.

Finally, note that if $a, b \in[1, \infty]$ and $c \in(0, \infty]$, the inclusion

$$
F L^{a} \subseteq F L^{b}+Z^{c}
$$

is equivalent to

$$
L^{a} \subseteq L^{b}+P F^{f}
$$

the sums on the right being vectorial. We shall often make the type of interchange exemplified by (4.2) and (4.3) without special comment.

There will be occasion to consider $L^{r} \cap P M^{q}$. When $r \geq 1$, this is interpreted by regarding both $L^{r}$ and $P M^{q}$ as subsets of $P M$ (more strictly, $L^{r}$ is identified witn its image in $\left.P M\right)$. If $0<r<1$, however, there is no natural injection of $L^{r}$ into $P M$ and no suitable interpretation of $L^{r} \cap P M^{q}$. (A literal interpretation of this intersection would make it $\emptyset$.)

THEOREM 4.2. (i) Suppose that $p, r \in[1, \infty]$ and $q \in(0, \infty]$. In order that $(p, r ; q)$ be admissible, it is necessary and sufficient that

$$
L^{r} \cap P M^{q} \subseteq L^{p}
$$

(ii) Suppose that $p, r \in[1, \infty)$ and $q \in[1, \infty]$. In order that $(p, r ; q)$ be admissible, it is necessary and sufficient that

$$
F L^{p^{\prime}} \subseteq F L^{r^{\prime}}+\tau^{q^{\prime}}
$$

that is, that

$$
L^{p^{\prime}} \subseteq L^{r^{\prime}}+P M^{q^{\prime}}
$$

Proof. (i) If $(p, r ; q)$ is admissible we have, for a suitable positive real number $B$, the inequality

$$
\|f\|_{p} \leq B\left(\|f\|_{p}+\|\hat{f}\|_{q}\right)
$$

for every $f \in T P$. Let $f \in L^{r} \cap B M^{q}$ and let $\left(K_{j}\right)$ be an approximate identity of trigonometric polynomials such that $\left\|K_{j}\right\|_{1} \leq 1$ for every $j$. Putting $f_{j}=K_{j} * f$, we then have 


$$
\left\|f_{j}-f\right\|_{p} \rightarrow 0 \text { and }\left\|\hat{f}_{j}-\hat{f}\right\|_{q} \rightarrow 0
$$

as $j$ increases. Applying (4.7) with $f$ replaced by $f_{j}-f_{k}$, it follows that $\left(f_{j}\right)$ is a Cauchy net in $L^{p}$ and so converges in $L^{p}$ to some $g \in L^{p}$. As a consequence, $\hat{f}_{j} \rightarrow \hat{g}$ pointwise on $X$. Since also $\hat{f}_{j} \rightarrow \hat{f}$ pointwise on $X$, it follows that $\hat{f}=\hat{g}$ and hence $f=g \in L^{p}$, showing that $(4.4)$ holds.

Conversely, suppose that $(4.4)$ holds. Regard $E=L^{r} \cap P M^{q}$ as a complete metrisable topological linear space (with the weakest topology making the injection maps of $E$ into $L^{r}$ and into $E M^{q}$ continuous). By hypothesis, the function $v: f \leftrightarrow\|f\|_{p}$ is finite-valued on $E$. It is easy to check (using Fatou's Lemma) that $v$ is lower semicontinuous on $E$. So, by Baire's Theorem, $v$ is bounded on some nonvoid open subset of $E$. This signifies the existence of $f_{0} \in E$ and positive real numbers $d$ and $m$ such that the conditions

$$
f \in E \text { and } \max \left(\left\|f-f_{0}\right\|_{p},\left\|\hat{f}-\hat{f}_{0}\right\|_{q}\right) \leq d
$$

together imply that $\|f\|_{p} \leq m$. Putting $m^{\prime}=m+\left\|f_{O}\right\|_{p}$, it then follows easily that (4.7) holds, with $B=m^{\prime} d^{-1}$, for every $f \in T P$. Thus $(p, r ; q)$ is admissible.

This completes the proof of (i).

(ii) This is a consequence of the general Lemma 4.8 below, applied with $X=T P$ taken with the $A$-norm; $Y=T P$ taken with the ${ }_{L}^{P}$-norm, $T$ the injection of $X$ into $Y ; Y_{1}=L^{r}, T_{1}$ the injection of $X$ into $y_{1} ; y_{2}=2^{q}$ if $q \neq \infty$ or $c_{0}$ if $q=\infty$, taken with the $2^{q}$-norm in either case, and $T_{2}$ the Fourier transformation. $X^{\prime}$ is identified with $P M ; Y^{\prime}$ and $Y_{1}^{\prime}$ are identified with $L^{P^{\prime}}$ and $L^{P^{\prime}}$ in the usual way, the coupling being expressed by $\langle f, g\rangle=f \star g(e) ;$ and $Y_{2}^{\prime}$ is 
identified with $\tau^{q^{\prime}}$ in all cases. Admissibility of $(p, r ; q)$ signifies that Lemma 4.8 (i) holds. On the other hand, in view of (4.1), Lemma 4.8 (ii) signifies that to every $g \in L^{p^{\prime}}$ correspond $h \in L^{r^{\prime}}$ and $\psi \in 2^{q^{\prime}}$ such that

$$
f * g(e)=f * h(e)+\sum_{x \in X} \psi(x) \hat{f}(x)
$$

for every $f \in T P$. This last equality signifies that

$$
\hat{g}=\hat{h}=\psi \text {. }
$$

Thus Lemma 4.8 (ii) signifies that (4.5) holds and the proof is complete.

COROLLARY 4.3. Suppose that $p \in(1, \infty)$ and $q \in[1, \infty]$. In order that $(p, q)$ be admissibie, it is necessary that

$$
F L^{p^{\prime}} \subseteq F L^{r^{\prime}}+Z^{q^{\prime}} \text {, that is, } L^{p^{\prime}} \subseteq L^{r^{\prime}}=P M^{q^{\prime}}
$$

for every $r \in[1, p)$, and sufficient that (4.8) be true for at least one $r \in[1, p)$.

Proof. This follows on combining (ii) of Theorem 4.2 with Lemma 1.4 and Definition 1.5.

REMARKS 4.4. On combining Corollary 4.3 with Theorem 2.1 we infer that, if $p \in(1, \infty), r \in[1, p]$ and $q \in(2, \infty]$, then

$$
F L^{p^{\prime}} \pm F L^{r^{\prime}}+Z^{q^{\prime}} \text {, that is, } L^{p^{\prime}} \pm L^{r^{\prime}}+P M^{q^{\prime}} \text {. }
$$

Likewise, from Corollary 4.3 combined with Theorem 3.1 it follows that, if $q \in(1,2], p \in\left(q^{\prime}, \infty\right)$ and $r \in[1, p)$, then $(4.9)$ is again true.

Replacing $p^{\prime}, r^{\prime}$ and $q^{\prime}$ by $a, b$ and $c$ respectively, (4.9) reads

$$
\text { (4.10) } F L^{a} \notin F L^{b}+l^{c} \text {, that is, } L^{a} \pm L^{b}+P M^{c} \text {, }
$$

which relations are therefore true if either

$$
a \in[1, \infty), b \in(a, \infty), c \in[1,2)
$$

or

$$
c \in[2, \infty), \quad a \in\left[1, c^{\prime}\right), b \in(a, \infty]
$$


(The condition $p \in(1, \infty)$ is equivalent to $a \in(1, \infty)$; clearly, if (4.10) holds for $a \in(1, \infty)$ or for $a \in\left(1, c^{\prime}\right)$, then it also holds for $a \in[1, \infty)$ or for $\left.a \in\left[1, c^{\prime}\right).\right)$

By using some general theorems from functional analysis, these inclusion results can be sharpened.

THEOREM 4.5. (i) If $a \in[1, \infty)$, then

(4.13) $F L^{a} \pm \underset{b>a}{U} F L^{b}+\underset{c<2}{U} l^{c}$, that is, $L^{a} \pm \underset{b>a}{U} L^{b}+\underset{c<2}{U} P M^{c}$.

(ii) If $b \in(1, \infty]$, then

(4.14) $\prod_{1 \leq a<b} F L^{a} \pm F L^{b}+\underset{c<2}{U} \tau^{c}$, that is, $\prod_{1 \leq a<b}^{n} L^{a} \pm L^{b}+\underset{c<2}{U} P M^{c}$.

Proof. (i) Take sequences $\left(b_{n}\right)$ and $\left(c_{n}\right)$ such that

$$
b_{n}>a, b_{n}+a, \quad 1 \leq c_{n}<2, c_{n} \uparrow 2 \text {. }
$$

Then

$$
\bigcup_{b>a} F L^{b}+\underset{c<2}{U} Z^{c}=U_{n}\left(F L^{b}+z^{c} n\right) .
$$

Supposing (4.13) to be false, we should therefore have

$$
F L^{a} \subseteq U_{n}\left(F L^{b}+L^{c} n\right)
$$

Now apply Theorem 6.5.1 of [1] (with $F=L^{a} ; u: f \mapsto \hat{f}$;

$F_{n}=L^{b} n \times 2^{c} n ; u_{n}:(g, \phi) \leftrightarrow \hat{g}+\phi ; E=C^{N}$ with the product topology,

$C$ denoting the complex field and $N$ the set of positive integers) to conclude that there exists $n$ for which

$$
E L^{a} \subseteq F L^{b}+2^{c} n \text {. }
$$

Since $b_{n}>a$ and $1 \leq c_{n}<2,(4.16)$ contradicts $(4.10)$ in the case specified by $(4.11)$.

(ii) Take a sequence $\left(a_{n}\right)$ such that $1 \leq a_{n}<b$ and $a_{n} \uparrow b$; let 
$\left(c_{n}\right)$ be as in (i) above. If (4.14) were false, we should have

$$
\prod_{n} F L^{a} \subseteq E L^{b}+U_{n} i^{c} n=\underset{n}{U}\left(E L^{b}+2^{c} n\right)
$$

Apply Theorem 6.5.1 of [2] (this time taking $F=\prod_{n} F L^{a_{n}}$ with the weakest topology making all the injections $F \rightarrow E L{ }^{a}$ continuous; $u: f \mapsto \hat{f}$; $F_{n}=L^{b} \times \tau^{c} n ; u_{n}:(g, \phi) \mapsto \hat{g}+\phi ; E=\mathrm{C}^{\mathrm{N}}$ with the product topology) to conclude the existence of a positive integer $m$ such that

$$
\bigcap_{n}{ }^{a} L^{n} \subseteq F L^{b}+2^{c} m
$$

Now apply Lemma 4.9 below, taking therein $E=i^{\infty}, E_{j}=L^{a}$, $F=F_{1}, \quad H=L^{b} \times \imath^{c} m, s: f \mapsto \hat{f}, \quad t:(g, \phi) \mapsto \hat{g}+\phi$. Using the fact that the closed unit ball in $H$ is compact for the product of the weak topologies $\sigma\left(L^{b}, L^{b^{\prime}}\right)$ and $\sigma\left(\tau^{c}, \tau^{c^{\prime}}\right)$ it is easy to check that Lemma 4.9 ( $i v)$ is satisfied; notice that $t$ is continuous for $\sigma\left(L^{b}, L^{b^{\prime}}\right) \times \sigma\left(\tau^{c} m, Z^{c^{\prime}}\right)$ on $H$ and the product topology on $E$ as a subset of $C^{N}$. All the other hypotheses of Lemma 4.9 are obviously fulfilled, Lemma 4.9 (v) being a reformulation of (4.18). We thus conclude that there exists a positive integer $j$ such that

$$
F L^{a} \subseteq F L^{b}+\imath^{c} m
$$

However, since $1 \leq a_{j}<b$ and $1 \leq c_{m}<2$, this again contradicts (4.10) in the case specified by (4.11).

THEOREM 4.6. If $a \in[1,2)$, then (4.19) $F L^{a} \pm \underset{b>a}{\mathrm{U}} F L^{b}+\underset{c<a^{\prime}}{\mathrm{U}} z^{c}$, that is, $L^{a} \pm \underset{b>a}{\mathrm{U}} L^{b}+\underset{c<a^{\prime}}{\mathrm{U}} P M^{\mathcal{F}}$. 
Proof. This proceeds in the same manner as does that of Theorem 4.5 (i), taking sequences $\left(b_{n}\right)$ and $\left(c_{n}\right)$ such that $b_{n}>a, b_{n}+a$, $2 \leq c_{n}<a^{\prime}, c_{n} \uparrow a^{\prime}$, noting that the negation of (4.19) implies that

$$
F L^{a} \subseteq U_{n}\left(F L^{b}+z^{c} n\right),
$$

and then applying Theorem 6.5.1 of [1] to reach a contradiction of (4.10) in the case specified by (4.12).

REMARK 4.7. The Hausdorff-Young theorem for $G$ implies that $L^{a} \subseteq P M^{a^{\prime}}$ whenever $a \in[1,2]$. Compare this with (4.19), noting that in the latter $a^{\prime}$ is just greater than $c$ if $a$ is just less than $c^{\prime}$. Note also that when $c>2, P M^{f}$ contains true pseudomeasures (that is, pseudomeasures which are not measures).

LEMMA 4.8. Let $X$ be a topological linear space, and $Y, Y_{1}, \ldots, Y_{n}$ normed linear spaces. Let $T$ be a continuous linear mapping of $X$ into $Y, T^{\prime}: Y^{\prime} \rightarrow X^{\prime}$ its adjoint; and, for each $k \in\{1,2, \ldots, n\}$, let $T_{k}$ be a continuous linear mapping of $X$ into $Y_{k}, T_{k}^{\prime}: Y_{k}^{\prime}+X^{\prime}$ its adjoint. The folzowing two assertions are equivalent:

(i) there exists a positive real number $B$ such that

$$
\|T x\| \leq B \cdot \sum_{k=1}^{n}\left\|T_{k} x\right\|
$$

$$
\text { for every } x \in X \text {; }
$$

$$
\text { (ii) } T^{\prime}\left(Y^{\prime}\right) \subseteq \sum_{k=1}^{n} T_{k}^{\prime}\left(Y_{k}^{\prime}\right) \text {. }
$$

(Compare Exercise 8.36 in [1].)

Proof. We first show that (i) implies (ii). Assuming (i), if $y^{\prime} \in Y^{\prime}$ we have for every $x \in X$

$$
\left|y^{\prime}(T x)\right| \leq \text { const. } \sum_{k=1}^{n} \| T k^{x \|} .
$$


Accordingly, there is a continuous linear functional $L$ with domain the linear subspace $\left\{\left(T_{k} x\right)_{1 \leq k \leq n}: x \in X\right\}$ of $y_{1} \times \ldots \times y_{n}$ which maps $\left(T_{k} x\right)_{1 \leq k \leq n}$ into $y^{\prime}(T x)$. By the Hahn-Banach Theorem, combined with the known form of the dual of $y_{1} \times \ldots \times y_{n}$ (see [1], Exercise 2.18), it follows that there exists $\left(y_{k}^{\prime}\right)_{I \leq k \leq n} \in Y_{I}^{\prime} \times \ldots \times Y_{n}^{\prime}$ such that the linear functional

$$
\left(y_{k}\right)_{1 \leq k \leq n} \mapsto \sum_{k=1}^{n} y_{k}^{\prime}\left(y_{k}\right)
$$

extends $L$. Then we have for every $\approx \in X$ the formula

$$
y^{\prime}(T x)=\sum_{k=1}^{n} y_{k}^{\prime}\left(T_{k} x\right)=\sum_{k=1}^{n}\left(T_{k}^{\prime} y_{k}^{\prime}\right)(x),
$$

which shows that

$$
T^{\prime} y^{\prime}=\sum_{k=1}^{n} T_{k}^{\prime} y_{k}^{\prime}
$$

and so proves that ( $i i)$ is satisfied.

To prove the converse, assume that (ii) is true and write $A$ for the set of $y^{\prime} \in y^{\prime}$ such that

$$
T^{\prime} y^{\prime} \in \sum_{k=1}^{n} T_{k}^{\prime}\left(v_{k}\right)
$$

where $U_{k}$ denotes the closed unit ball in $Y_{k}^{\prime}$. By hypothesis, $A$ is absorbent in $Y^{\prime}$. On the other hand, $A$ is plainly convex and balanced. Since also $T_{k}^{\prime}$ is weakly continuous (that is, continuous for $\sigma\left(Y_{k}^{\prime}, Y_{k}\right)$ and $\left.\sigma\left(X^{\prime}, X\right)\right)$ and $i_{k}$ is weakly compact, $\sum_{k=1}^{n} T_{k}^{\prime}\left(U_{k}\right)$ is weakly compact, hence weakly closed. Since $T^{\prime}$ is weakly continuous, $A$ is weakly closed, hence norm-closed in $Y^{\prime}$. Thus $A$ is a barrel in $Y^{\prime}$ and therefore a neighbourhood of 0 in $Y^{\prime}$. In other words, there is a positive real number $B$ such that for every $y^{\prime} \in Y^{\prime}, T^{\prime} y^{\prime}$ is representable as in (4.21) with 


$$
\left\|y_{k}^{\prime}\right\| \leq B\left\|y^{\prime}\right\| \text { for every } k \in\{1,2, \ldots, n\} \text {. }
$$

This being so, let $x \in X, y^{\prime} \in Y^{\prime}$ and $\left\|y^{\prime}\right\| \leq 1$. Choose the $y_{k}^{\prime}$ so that (4.21) and (4.22) hold. We then have

$$
\begin{aligned}
\left|y^{\prime}(T x)\right|=\left|T^{\prime} y^{\prime}(x)\right| & =\left|\sum_{k=1}^{n}\left(T_{k}^{\prime} y_{k}^{\prime}\right)(x)\right| \\
& \leq \sum_{k=1}^{n}\left|y_{k}^{\prime}\left(T_{k} x\right)\right| \\
& \leq \sum_{k=1}^{n}\left\|y_{k}^{\prime}\right\| \cdot\left\|T_{k} x\right\| \\
& \leq B \sum_{k=1}^{n}\left\|T_{k} x\right\| .
\end{aligned}
$$

Letting $y^{\prime}$ vary, (4.20) follows.

LEMMA 4.9. Suppose that

(i) $E$ and $F$ are topological linear spaces, $H$ a normed linear space with closed unit ball $U$;

(ii) $\left(E_{m}\right)$ is a sequence of linear subspaces of $F$, each a Fréchet space with some topology, $F_{m+1} \subseteq F_{m}$, the injections $F_{m+1} \rightarrow F_{m}$ and $F_{m} \rightarrow F$ being continuous;

(iii) $s$ is a continuous linear map from $F$ into $E$ and $t a$ linear map from $H$ into $E$;

(iv) $A=t(U)$ is closed in $E$;

(v) $s\left(\prod_{m=1}^{\infty} F_{m}\right) \subseteq t(H)$;

(vi) $\prod_{m=1}^{\infty} F_{m}$ is dense in $F_{n}$ for every $n$.

The conclusion is that there exist a positive integer $n$ and a.continuous seminorm $p$ on $F_{n}$ such that

(vii) $s(y) \subseteq(\varepsilon+p(y)) t(U)$ for every $y \in F_{n}$ and every $\varepsilon>0$; in 
particuzar, $s\left(F_{n}\right) \subseteq t(H)$.

Proof. Form $P=\bigcap_{m=1}^{\infty} F_{m}$ into a Fréchet space with the weakest topology such that all the injections $P \rightarrow F_{m}$ are continuous. Define

$$
S=\{y \in P: s(y) \in A\}=P \cap s^{-1}(A) \text {. }
$$

$S$ is plainly convex and balanced; it is closed in $P$ because of ( $i i)$, ( $i$ ii) and ( $i v)$; and $i t$ is absorbent because of ( $v$ ). $S$ is therefore a neighbourhood of zero in $P$. This means that there exist a positive integer $n$ and a continuous seminorm $p$ on $F_{n}$ such that to every $y \in P$ corresponds $z \in H$ such that $s(y)=t(z)$ and $\|z\| \leq p(y)$.

Now let $y \in F_{n}$. By $(v i)$, there exists a sequence $\left(y_{j}\right)$ of elements of $P$ converging in $F_{n}$ to $y$. Then $p\left(y_{j}\right) \rightarrow p(y)$. By what has just been established, to every $j$ corresponds $z_{j} \in H$ such that

$$
\left\|z_{j}\right\| \leq p\left(y_{j}\right) \text { and } s\left(y_{j}\right)=t\left(z_{j}\right) \text {. }
$$

Taking any $k>p(y)$, we have $p\left(y_{j}\right) \leq k$ for every $j \geq j_{0}$. For such $j,\left\|k^{-1} z_{j}\right\| \leq 1$ and so

$$
s\left(k^{-1} y_{j}\right) \in A
$$

Using (ii), (iii) and (iv) it follows that $y_{j} \rightarrow y$ in $F$ and so

$$
s\left(k^{-1} y\right)=E-\operatorname{lims}\left(k^{-1} y_{j}\right) \in A
$$

Hence

$$
s(y) \in k A=k t(U) .
$$

This is equivalent to (vii) and the proof is complete.

REMARK 4.10. Lemma 4.9 (vii) implies that

$$
s(y) \subseteq(1+\varepsilon) p(y) \cdot t(U)
$$

for every $\varepsilon>0$ and every $y \in F_{n}$ satisfying $p(y) \neq 0$. The restriction $p(y) \neq 0$ can be removed if either 
(a) there exists on $F_{n}$ a continuous norm, or

(b) $E$ is Hausdorff and $t(U)=A$ is bounded in $E$ (which is so whenever $t$ is continuous from $H$ into $E$ ).

In fact, if (a) holds, we may assume that $p$ is a norm on $F_{n}$, so that $p(y) \neq 0$ whenever $y \neq 0$; and (4.23) is in any case trivially true whenever $s(y)=0$, and so, in particular, whenever $y=0$. If, on the other hand, $A$ is bounded in $E$, and if $p(y)=0$, Lemma 4.9 (vii) implies that $s(y)$ belongs to the closure in $E$ of $\{0\}$; if $E$ is Hausdorff, this entails that $s(y)=0$ and so that (4.23) is trivially true.

\section{A constructional procedure}

Suppose it to be known that $p \in(1, \infty], q \in(0, \infty]$ and that $(p, q)$ is not admissible ( $c f$. Theorems 2.1 and 3.1). Then, by Theorem 4.2 (i), we have

$$
L^{r} \cap P M^{q} \notin L^{p}
$$

for every $r \in(0, p)$. An appeal to Lemma 4.9 will show that (5.1) implies that

$$
\left(\bigcap_{r<p} L^{r}\right) \cap P M^{q} \pm L^{p},
$$

though of course the lemma does not indicate how to find functions $f$ satisfying

$$
f \in\left(\bigcap_{r<p} L^{r}\right) \cap P M^{q}, f \notin L^{p} .
$$

It is however possible to construct such functions $f$ fairly explicitly. To do this, choose a sequence $\left(r_{n}\right)$ from $(0, p)$ such that $r_{n} \uparrow p$. Since $\left(p, r_{n} ; q\right)$ is not admissible, there are trigonometric polynomials $f_{n}$ such that

$$
\left\|f_{n}\right\|_{p}>n\left(\left\|f_{n}\right\|_{r_{n}}+\left\|\hat{f}_{n}\right\|_{q}\right) .
$$


In the cases covered by Theorem 2.1, such a sequence $\left(f_{n}\right)$ may be taken as a subsequence $\left(K_{j} * \mu\right)$ of $\left(K_{j} * \mu\right)$, where $\left(K_{j}\right)$ is an approximate identity of trigonometric polynomials and $\left(j_{n}\right)$ is any sequence of positive integers which tends to infinity sufficiently rapidly. In the cases covered by Theorem 3.1, we proceed likewise, $\mu$ being replaced by the function $f$ or $f^{\prime}$ appearing in (a), (b) or (c) of 3.3. In any case, write

$$
g_{n}=n^{-1 / 2}\left(\left\|f_{n}\right\|_{r_{n}}+\left\|\hat{f}_{n}\right\|_{q}\right)^{-1} f_{n}
$$

so that $g_{n} \in T P$ and

$$
\left\|g_{n}\right\|_{r_{n}} \leq n^{-1 / 2},\left\|\hat{g}_{n}\right\|_{p} \leq n^{-1 / 2},\left\|g_{n}\right\|_{p}>n^{1 / 2}
$$

Consider the first countable complete topological linear space

$$
\begin{aligned}
E & =\left(\bigcap_{r<p} L^{r}\right) \cap P M^{q} \\
& =\left(\cap L^{r} n\right) \cap P M^{q},
\end{aligned}
$$

the topology being the weakest making continuous all the injections $E \rightarrow L^{r}$ and $E \rightarrow P M^{q}$, and the gauges $F_{n}$ on $E$ defined by

$$
F_{n}(g)=\|\min (|g|, n)\|_{p}=\left(\int_{G}(\min (|g|, n))^{p} d \lambda\right)^{1 / p} .
$$

If $F^{*}$ is the upper envelope of the $F_{n}$, Fatou's Lemma shows that

$$
\left.F^{*}(g)=\iint_{G}^{*}|g|^{p} d \lambda\right)^{I / p} .
$$

Thus, $F^{*}\left(g_{n}\right)$ is finite for every $n$. Also, (5.5) shows that $g_{n} \rightarrow 0$ in $E$ and $F^{*}\left(g_{n}\right)>n^{1 / 2}$. Positive integers $m_{n}$ can therefore be found such that 


$$
F_{n}\left(g_{n}\right)>n^{1 / 2}
$$

Now apply Theorem 2.1 of [3] to $E$ and to the gauges $E_{m_{n}}$ to obtain sequences $n_{1}<n_{2}<\ldots$ of positive integers and elements

$$
f=g_{n_{1}}+g_{n_{2}}+\ldots
$$

of $E$ satisfying

$$
\lim _{k \rightarrow \infty} F_{m_{n_{k}}}(f)=\infty
$$

Reference to (5.6) and the definitions of $E$ and $F^{*}$ show that $f$ satisfies $(5.3)$.

\section{References}

[1] R.E. Edwards, Functional analysis. Theory and applications (Holt, Rinehart and Winston, New York, Chicago, San Francisco, Toronto, London, 1965).

[2] R.E. Edwards, Fourier series: A modern introduction, Volumes I and II (Holt, Rinehart and Winston, New York, Chicago, San Francisco, Atlanta, Dallas, Montreal, Toronto, London, 1967).

[3] R.E. Edwards and J.F. Price "A naively constructive approach to boundedness principles, with applications to harmonic analysis", Enseignement Math. (2) 16 (1970), 255-296 (1971).

[4] Edwin Hewitt and Kenneth A. Ross, Abstract harmonic analysis, Volume I (Die Grundlehren der mathematischen Wissenschaften, Band 115. Academic Press, New York; Springer-Verlag, Berlin, Göttingen, Heidelberg, 1963). Abstract harmonic analysis, Volume II (Die Grundlehren der mathematischen Wissenschaften, Band 152. Springer-Verlag, Berlin, Heidelberg, New York, 1970). 
[5] Edwin Hewitt and Herbert S. Zuckerman, "Singular measures with absolutely continuous convolution squares", Proc. Cambridge Philos. Soc. 62 (1966), 399-420. "Singular measures with absolutely continuous convolution squares. (Corrigendum)", Proc. Combridge Philos. Soc. 63 (1967), 367-368.

[6] A. Zygmund, Trigonometric series, Volumes I and II, 2nd edition (Cambridge University Press, Cambridge, 1959; reprinted 1968).

Department of Mathematics, Institute of Advanced Studies, Australian National University, Canberra, ACT. 